\title{
Phosphatidylserine-exposing cells contribute to the hypercoagulable state in patients with multiple myeloma
}

\author{
LI GUO $^{1}$, DONGXIA TONG ${ }^{1}$, MUXIN YU $^{1}$, YAN ZHANG ${ }^{1}$, TAO LI $^{1}$, CHUNXU WANG $^{1}$, PENG ZHOU $^{2}$, \\ JIAQI JIN ${ }^{2}$, BAORONG LI ${ }^{3}$, YINGMIAO LIU ${ }^{3}$, RUIPENG LIU ${ }^{4}$, VALERIE A. NOVAKOVIC ${ }^{5}$, \\ ZENGXIANG DONG ${ }^{6}$, YE TIAN ${ }^{6}$, JUNJIE KOU ${ }^{4}$, YAYAN BI ${ }^{6}$, JIN ZHOU ${ }^{1}$ and JIALAN SHI ${ }^{1,5}$ \\ ${ }^{1}$ Department of Hematology, The First Hospital of Harbin Medical University, Harbin, Heilongjiang 150001; \\ ${ }^{2}$ Department of Neurosurgery, The Second Hospital of Harbin Medical University, Harbin, Heilongjiang 150086; \\ ${ }^{3}$ Department of Stomatology, The First Hospital of Harbin Medical University, Harbin, \\ Heilongjiang 150001; ${ }^{4}$ Department of Cardiology, The Second Hospital of Harbin Medical University, \\ Harbin, Heilongjiang 150086, P.R. China; ${ }^{5}$ Department of Research and Surgery, VA Boston Healthcare System, \\ Brigham and Women's Hospital, Harvard Medical School, Boston, MA 02132, USA; ${ }^{6}$ Department of Cardiology, \\ The First Hospital of Harbin Medical University, Harbin, Heilongjiang 150001, P.R. China
}

Received December 18, 2017; Accepted March 6, 2018

DOI: 10.3892/ijo.2018.4354

\begin{abstract}
Multiple myeloma (MM) is characterized by an increased incidence of thromboembolic events, particularly when treated with immunomodulatory drugs (IMiDs) in combination with dexamethasone. The optimal prophylactic strategy to prevent the hypercoagulable state of patients with MM is still debated. The aim of the current study was to investigate the definitive role of phosphatidylserine (PS) in supporting procoagulant activity (PCA) in patients with MM. Patients with MM (n=20) and healthy subjects $(n=15)$ were recruited for the present study. PS analyses were performed by flow cytometry and confocal microscopy. The PCA was evaluated by clotting time, purified coagulation complex assays and fibrin production assays. The percentage of $\mathrm{PS}^{+}$blood cells was significantly higher in patients with MM than in healthy subjects. Additionally, the patient serum induced more PS exposure on endothelial cells (ECs) in vitro than serum from healthy subjects. Isolated blood cells from patients with MM and ECs cultured with patient serum in vitro demonstrated significantly shortened coagulation time, greatly intrinsic/ extrinsic factor Xa generation and increased thrombin formation. In addition, the levels of $\mathrm{PS}^{+}$erythrocytes, platelets, leukocytes, and ECs incubated with IMiDs and dexamethasone were higher than with IMiDs alone. The findings support
\end{abstract}

Correspondence to: Dr Jialan Shi or Dr Jin Zhou, Department of Hematology, The First Hospital of Harbin Medical University, 23 Youzheng Street, Nangang, Harbin, Heilongjiang 150001, P.R. China

E-mail:shi73661@gmail.com

E-mail: jinzhou1956@163.com

Key words: multiple myeloma, immunomodulatory drugs, phosphatidylserine, blood cell, hypercoagulable state the hypothesis that increased PS exposure on blood cells and ECs participates in the hypercoagulable state in patients with MM. Thus, blocking PS may be a novel therapeutic target for the prevention of thrombosis in these patients.

\section{Introduction}

Venous thromboembolism (VTE) is a common complication in patients with multiple myeloma (MM), who can have up to a 28 -fold increase in risk of VTE $(1,2)$. The occurrence of thrombosis seriously impacts quality of life and increases mortality of patients with MM (3). Notably, the incidence of VTE in patients with MM treated with immunomodulatory drug (IMiD) monotherapy is 2-6\%, while dexamethasone (Dex)-containing regimens dramatically increase the risk of VTE up to $17-26 \%$ in individual clinical trials $(4,5)$. Previous studies have reported that patients with MM have several risk factors for VTE, including increased levels of factor VIII and von Willebrand factor, hypofibrinolysis and acquired activated protein $\mathrm{C}$ resistance (6-9). However, these molecular mechanisms are not sufficient to reflect the changes in plasma procoagulant components and there is limited research studying the cellular mechanism of VTE in patients with MM. In addition, the evidence to guide optimal selection of thromboprophylaxis when managing patients with MM is still lacking. Thus, it is important to investigate the precise molecular and cellular mechanism of the hypercoagulable state in these patients.

Phosphatidylserine (PS) is exposed by the action of scramblase on the cell's surface during biological processes such as apoptosis and cell activation (10). Once exposed on the outer membrane, PS acts as a catalytic surface for factor $\mathrm{Xa}$ and thrombin formation in the coagulation cascade (11). Our previous studies have demonstrated that PS expression on the surface of circulating cells is associated with a risk of developing venous thrombotic complications in various disorders, including nephrotic syndrome and 
acute promyelocytic leukemia $(12,13)$. Previous studies have demonstrated that peripheral blood cells and endothelial cells (ECs) can be injured or activated by high levels of monoclonal paraprotein and inflammatory cytokines circulating in the blood of patients with MM (14-18). However, relatively little is known about the extent to which PS is exposed on these cells, or whether $\mathrm{PS}^{+}$cells contribute to a hypercoagulable state in patients with MM. Although other studies reported that IMiDs with Dex increased PS exposure on monocyte and EC cell lines (19,20), the inhibition assays were not performed to clarify whether PS was responsible for cell-associated procoagulant activity (PCA). Furthermore, it is unclear whether IMiD-based regimens will further aggravate the amount of PS exposure on blood cells or ECs from patients with MM treated with patient serum. Thus, lactadherin, a superior PS probe (21), was used to detect the exposure of PS on cells and define whether there is an association between PS levels, cell-associated PCA and development of the hypercoagulable state in patients with MM.

The main objective of the current study was to evaluate the PCA of PS ${ }^{+}$blood cells and ECs and to assess a possible association with the development of venous thrombosis. Furthermore, the effect of treatment with IMiDs with or without Dex on PS exposure in blood cells and ECs was evaluated, and their relevance to PCA of these cells. Additionally, inhibition experiments were performed to evaluate the anticoagulant property of lactadherin. The results suggested that PS exposure on blood cells and ECs has a pivotal role in the hypercoagulability in patients with MM.

\section{Materials and methods}

Study subjects. The study included 20 newly diagnosed patients with MM (range 31-78, 45\% males) according to the standards of the International Myeloma Working Group who were admitted to the First Affiliated Hospital of Harbin Medical University between October 2016 and February 2017, and 15 healthy volunteers (range $28-75,46.67 \%$ males) (22). The exclusion criteria were a recent ( $<6$ months) thrombotic event, current anticoagulant therapy, and associated disease, including antiphospholipid syndrome, chronic renal disease, heart disease, malignant or systemic disease, diabetes, acute infection, immobilization, surgery, hereditary thrombophilia and hyperviscosity, among other conditions. Harbin Medical University Ethical Committee (Harbin, China) approved the study, and patients provided written, informed consent.

Reagents. Thalidomide (Tha), lenalidomide (Len) and Dex were purchased from Sigma-Aldrich (Merck KGaA, Darmstadt, Germany). Human umbilical vein cells (HUVECs), ECs medium, and poly-L-lysine were from ScienCell Research Laboratories, Inc. (San Diego, CA, USA). RPMI-1640 medium, fetal bovine serum (FBS) and bovine serum albumin (BSA) were obtained from Gibco (Thermo Fisher Scientific, Inc., Waltham, MA, USA). Alexa Fluor 488 or Alexa Fluor 647-conjugated lactadherin were prepared in our laboratory. Human factors (F) Va, VIIa, VIII, IXa, X, Xa, prothrombin, thrombin, fluorescein EGR-chloromethylketone and biotinylated EGR-chloromethylketone were all from Haematologic Technologies, Inc. (Burlington, VT, USA). Mouse anti-fibrin II chain (cat. no. NYBT2G1) was from
Accurate Chemical \& Scientific Corporation (Westbury, NY, USA). Isotype control antibody (cat. no. X0931) was from Dako (Agilent Technologies, Inc., Santa Clara, CA, USA). Fluorescein-maleimide, fluorescein isothiocyanatephalloidin, DAPI, Alexa 647-labeled isotype-matched control antibody (cat. no. MA5-18168) were from Molecular Probes (Invitrogen; Thermo Fisher Scientific, Inc.). Propidium iodide (PI) was obtained from Shanghai Dobio Biotech Co., Ltd. (Shanghai, China). Chromogenic substrates S-2765 and S-2238 were from Diapharma Group, Inc. (West Chester, OH, USA). Percoll was from GE Healthcare Life Sciences (Uppsala, Sweden). Tyrode's buffer containing $1 \mathrm{mM}$ HEPES was prepared in our laboratory and filtered through a $0.22-\mathrm{mm}$ syringe filter from EMD Millipore (Billerica, MA, USA).

Protein purification and labeling. Lactadherin was purified from bovine milk and labeled with Alexa Fluor 647 or Alexa Fluor 488 as described previously (12). The ratio of fluorescein to lactadherin was $1.2 / 1$ or $1.1 / 1$.

Preparation of blood cells. Blood was drawn prior to therapy with a 21-gauge needle and was collected into an anticoagulant tube containing $3.2 \%$ citrate $(5 \mathrm{ml}$; BD Biosciences, San Jose, CA, USA). Blood cell isolation was performed $\leq 30 \mathrm{~min}$ postblood collection. Following centrifuging at $200 \mathrm{x}$ g for $15 \mathrm{~min}$ at $20^{\circ} \mathrm{C}$, platelet-rich plasma (PRP) was aspirated carefully from the upper layer and erythrocytes were collected from the bottom layer of blood samples. Mixed peripheral blood leukocytes were isolated from the blood of study subjects using gradient centrifugation with $30 \%$ percoll and $68 \%$ percoll, according to the manufacturer's protocol. Erythrocytes, PRP, and leukocytes were analyzed immediately by flow cytometry and confocal microscopy following isolation. To prepare platelet-free plasma (PFP), PRP was centrifuged for $20 \mathrm{~min}$ at $1,500 \mathrm{x} \mathrm{g}$ at room temperature. For microparticle-depleted plasma (MDP) preparation, $600 \mu$ l of PFP was centrifuged for $30 \mathrm{~min}$ at $20,000 \mathrm{x} \mathrm{g}$ at $20^{\circ} \mathrm{C}$. The supernatant $(400 \mu \mathrm{l})$ was collected, snap-frozen in liquid nitrogen, and then stored at $-80^{\circ} \mathrm{C}$ prior to use.

Preparation of immunomodulatory drugs and Dex. Tha and Len were dissolved in dimethyl sulfoxide (DMSO; Sigma-Aldrich; Merck KGaA) and Dex was resuspended in $1 \mathrm{X}$ PBS. The drugs were stored at $-20^{\circ} \mathrm{C}$ prior to use. Tha or Len and Dex were diluted in culture medium to reach the final concentrations of 1.0 and $10 \mu \mathrm{M}$, respectively. The final concentration of DMSO was $0.01 \%$ in all experiments as preliminary experiments had demonstrated that DMSO concentrations of $0.01 \%$ had no significant effect on PS exposure on cells (data not shown).

HUVEC culture and reconstitution experiments. HUVECs were maintained in $\mathrm{EC}$ medium in poly-L-lysine-coated cell culture flasks at $37^{\circ} \mathrm{C}$ and $5 \% \mathrm{CO}_{2}$ in a humid environment. HUVECs were incubated in growth media containing $20 \%$ pooled serum obtained from patients with MM or healthy subjects at room temperature for $24 \mathrm{~h}$. Serum-cultured ECs were then treated with DMSO $(0.01 \%)$, Tal $(1.0 \mu \mathrm{M})$, Len $(1.0 \mu \mathrm{M})$, Tal $(1.0 \mu \mathrm{M}) /$ Dex $(10 \mu \mathrm{M})$, or Len $(1.0 \mu \mathrm{M}) / \operatorname{Dex}(10 \mu \mathrm{M})$ at room temperature for $24 \mathrm{~h}$. Cells in the logarithmic growth phase were used 
for all experiments. All cell culture results presented are based on at least three individual experiments.

Blood cells of incubation and reconstitution experiments. Erythrocytes, platelets and leukocytes from patients with MM were cultured separately with DMSO $(0.01 \%)$, Tal $(1.0 \mu \mathrm{M})$, Len $(1.0 \mu \mathrm{M}), \operatorname{Tal}(1.0 \mu \mathrm{M}) / \operatorname{Dex}(10 \mu \mathrm{M}), \operatorname{or} \operatorname{Len}(1.0 \mu \mathrm{M}) / \operatorname{Dex}(10 \mu \mathrm{M})$ in a $5 \% \mathrm{CO}_{2}$ atmosphere at $37^{\circ} \mathrm{C}$. Erythrocytes $\left(10^{6} / \mathrm{ml}\right)$ were incubated in vitro for $24 \mathrm{~h}$ at a hematocrit of $0.4 \%$ in Ringer's solution (23). Platelets $\left(10^{6} / \mathrm{ml}\right)$ were suspended in Tyrode's buffer for $1 \mathrm{~h}$ (24). Leukocytes $\left(10^{6} / \mathrm{ml}\right)$ were incubated for $24 \mathrm{~h}$ in RPMI-1640 medium containing 10\% FBS (25). Erythrocytes, platelets and leukocytes were washed twice before detection.

Flow cytometric analysis of PS exposure on blood cells and $E C s$. To quantify PS exposure on blood cells and ECs, $5 \mu 1$ of each cell suspension $\left(0.5-1 \times 10^{6} / \mathrm{ml}\right)$ in Tyrode's buffer was incubated with $5 \mu$ Alexa Fluor 488-conjugated lactadherin for $15 \mathrm{~min}$ at room temperature in the dark. A total of 10,000 events per sample were acquired by flow cytometry and analyzed with BD FACSDiva software 6.0 (BD Biosciences).

Clotting time and inhibition assays. PCA of various cell types was evaluated using a one-stage recalcification time assay in a KC4A-coagulometer (Amelung; Labcon GmbH, Heppenheim, Germany). Cell suspensions [100 $\mu 1$ of erythrocyte $\left(1 \times 10^{8}\right)$, platelet $\left(1 \times 10^{7}\right)$, leukocyte $\left(1 \times 10^{6}\right)$, or EC $\left.\left(1 \times 10^{6}\right)\right]$ were incubated with $100 \mu \mathrm{l} \mathrm{MDP}$ from healthy volunteers at $37^{\circ} \mathrm{C}$. After $3 \mathrm{~min}, 100 \mu \mathrm{l}$ of warmed $25 \mathrm{mM} \mathrm{CaCl}_{2}$ was added to start the reaction, and the time to subsequent fibrin strand formation was recorded. All clotting assays were performed in triplicate. For inhibition assays, cells were preincubated with lactadherin (final concentration $128 \mathrm{nM}$ ) prior to the assay.

Factor Xa and prothrombinase formation and inhibition assay. For the intrinsic Xa formation, a total of $10^{4}$ platelets/leukocytes/ECs or $10^{5}$ erythrocytes were incubated with $1 \mathrm{nM}$ factor IXa, $5 \mathrm{nM}$ factor VIII, $0.2 \mathrm{nM}$ thrombin, $130 \mathrm{nM}$ factor $\mathrm{X}$, and $5 \mathrm{mM} \mathrm{CaCl}_{2}$ in factor Xa buffer (TBS with $0.2 \% \mathrm{BSA}$ ) for $5 \mathrm{~min}$ at $20^{\circ} \mathrm{C}$. The reaction was stopped by the addition of EDTA ( $7 \mathrm{mM}$ final concentration). Immediately following the addition of $10 \mu \mathrm{l} \mathrm{S}-2765(0.8 \mathrm{mM})$ chromogenic substrate, factor Xa generation was determined using a kinetic absorbance reading at $405 \mathrm{~nm}$ on a SpectraMax M5 Microplate Reader (Molecular Devices, LLC, Sunnyvale, CA, USA). The assay to measure formation of extrinsic factor Xa was analogous to that for intrinsic factor $\mathrm{Xa}$ except that cells were mixed with $1 \mathrm{nM}$ factor VIIa, $130 \mathrm{nM}$ factor $\mathrm{X}$ and $5 \mathrm{mM} \mathrm{CaCl}_{2}$. For the prothrombinase assay, the samples were incubated with $1 \mathrm{nM}$ factor $\mathrm{Va}, 2 \mathrm{nM}$ factor $\mathrm{Xa}, 1 \mu \mathrm{M}$ prothrombin and $5 \mathrm{mM} \mathrm{CaCl}_{2}$ in prothrombinase buffer (TBS with $0.05 \%$ BSA) for 5 min at $25^{\circ} \mathrm{C}$. Thrombin production was evaluated after the addition of EDTA using the chromogenic substrate S-2238 (0.8 mM). For the inhibition assays of intrinsic/extrinsic factor $\mathrm{Xa}$ and prothrombinase formation, cells suspensions were pre-incubated with lactadherin (final concentration $128 \mathrm{nM}$ ) for $10 \mathrm{~min}$ at $25^{\circ} \mathrm{C}$ in Tyrode's buffer. The mixture was then incubated with the specified clotting factors according to the above protocols. The quantity of thrombin or factor Xa formation was assessed as previously described (12).
Fibrin generation assay. Fibrin formation was quantified by turbidity as described (26). For assays in normal human MDP, blood cells and cultured ECs were added to recalcified $(10 \mathrm{mM}$, final) normal human MDP ( $88 \%$ MDP, final) in the absence or presence of lactadherin (final concentration $128 \mathrm{nM}$ ). Fibrin production was measured by turbidity at $405 \mathrm{~nm}$ in a SpectraMax 340PC plate reader (Molecular Devices).

Confocal microscopy. To locate PS, erythrocytes, platelets or leukocytes were incubated with Alexa Fluor 488-lactadherin (final concentration $4 \mathrm{nM}$ ) or PI, and ECs were incubated with the final concentrations of $4 \mathrm{nM}$ Alexa Fluor 488-lactadherin and Alexa Fluor 647-CD31 [purified CD31, (clone L133.1), $\mathrm{BD}$ Biosciences)] for $10 \mathrm{~min}$ at room temperature in the dark. Then, cells were washed to remove unbound dye and imaged immediately. Observation of PS exposure on cells by confocal microscopy was performed as previously described (12). For fibrin generation experiments in vitro, ECs were cultured on $1 \%$ gelatin-coated coverslips in media containing $20 \%$ normal or patient serum for $24 \mathrm{~h}$. Following rinsing with Tyrode's buffer, the ECs were then overlaid with prewarmed MDP (15\%) in the presence of $3 \mathrm{mM}$ calcium. Fibrin networks were imaged using laser confocal microscopy in the presence of Alexa Fluor 647-conjugated anti-fibrin (final concentration, $1 \mu \mathrm{g} / \mathrm{ml}$; prepared in our laboratory) at $37^{\circ} \mathrm{C}$ for $15 \mathrm{~min}$. Background signal was calculated using a similarly labeled isotype matched control antibody (final $1 \mu \mathrm{g} / \mathrm{ml}$ ) at $37^{\circ} \mathrm{C}$ for $15 \mathrm{~min}$. To observe the location of coagulation factor binding sites, the ECs were co-stained with factor Va-fluorescein-maleimide (final concentration, $2 \mathrm{nM}$ ) and factor Xa-EGRck-biotin (final concentration, $2 \mathrm{nM}$, complexed to Alexa Fluor 647-streptavidin) at $37^{\circ} \mathrm{C}$ for $10 \mathrm{~min}$. Samples were excited with 488 or $568 \mathrm{~nm}$ emission lines of a krypton-argon laser and narrow bandpass filters were used for restricting emission wavelength overlap. Images were obtained using the LSM 510 system (Carl Zeiss AG, Oberkochen, Germany).

Statistical analysis. Results are presented as mean \pm standard deviation of at least triplicate measurements. Statistical analysis was performed with Student's t-test or one-way analysis of variance followed by Fisher's least significant difference post hoc tests for multiple comparisons as appropriate. $\mathrm{P}<0.05$ was considered to indicate a statistically significant difference.

\section{Results}

Patient characteristics. The characteristics of the study participants (20 patients with MM and 15 healthy subjects) are detailed in Table I. Patients with MM had a high serum concentration of M-component. Compared with healthy subjects, patients with MM had significantly higher levels of $\beta 2$-microglobulin, fibrinogen, D-dimer, von Willebrand factor and factor VIII and shortened prothrombin time. However, patients with MM had lower levels of erythrocytes and hemoglobin than healthy subjects.

PS exposure of blood cells from healthy subjects and patients with MM patients, and in cultured ECs. As lactadherin binds 
Table I. Clinical characteristics of healthy subjects and patients with multiple myeloma.

\begin{tabular}{|c|c|c|}
\hline Characteristic & Healthy subjects & MM \\
\hline Total n & 15 & 20 \\
\hline Age (years) & $57 \pm 7$ & $60 \pm 11$ \\
\hline Male, n (\%) & $7(46.67 \%)$ & $9(45 \%)$ \\
\hline International Staging System stage I/II/III, n (\%) & NA & $4 / 7 / 9(20 \% / 35 \% / 45 \%)$ \\
\hline \multicolumn{3}{|l|}{ M-protein class, $\mathrm{n}(\%)$} \\
\hline $\operatorname{IgG}$ & NA & $14(70 \%)$ \\
\hline $\operatorname{IgA}$ & NA & $6(30 \%)$ \\
\hline Serum M protein $(\mathrm{g} / \mathrm{l})$ & NA & $25 \pm 12$ \\
\hline$\beta 2$-microglobulin (mg/l) & $1.65 \pm 0.55$ & $6.88 \pm 5.08^{\mathrm{a}}$ \\
\hline Erythrocytes $\left(\times 10^{12} / 1\right)$ & $4.10 \pm 0.44$ & $3.31 \pm 1.01^{\mathrm{a}}$ \\
\hline Platelet (x10 $/ 1)$ & $228 \pm 25$ & $188 \pm 82$ \\
\hline Leukocytes (x 109/1) & $5.79 \pm 0.63$ & $5.31 \pm 1.38$ \\
\hline Albumin (g/l) & $42.4 \pm 3.6$ & $37.6 \pm 7.4$ \\
\hline Hemoglobin $(g / 1)$ & $124 \pm 8$ & $101 \pm 29^{a}$ \\
\hline Prothrombin time (sec) & $13.48 \pm 0.76$ & $12.52 \pm 1.04^{\mathrm{a}}$ \\
\hline Activated partial thromboplastin time (sec) & $33.9 \pm 2.0$ & $31.6 \pm 4.5$ \\
\hline Fibrinogen $(\mathrm{g} / \mathrm{l})$ & $2.61 \pm 0.32$ & $3.07 \pm 0.96$ \\
\hline D-dimer (mg/l) & $0.20 \pm 0.07$ & $1.81 \pm 1.26^{\mathrm{a}}$ \\
\hline Von Willebrand factor (U/ml) & $1.06 \pm 0.26$ & $2.23 \pm 0.71^{\mathrm{a}}$ \\
\hline Factor VIII (U/ml) & $0.87 \pm 0.18$ & $2.10 \pm 0.62^{\mathrm{a}}$ \\
\hline
\end{tabular}

${ }^{a} \mathrm{P}<0.05$ vs. healthy subjects. NA, not applicable. Data are presented as numbers (percentages) or mean \pm standard deviation.

to PS with high affinity (21), fluorescence-labeled was used lactadherin to detect the level of PS exposure on various blood cells using a flow cytometer. Patients with MM had a significantly higher percentage of $\mathrm{PS}^{+}$blood cells than healthy subjects (RBC, $1.12 \pm 0.58$ vs. $0.54 \pm 0.29 \%$; PLT, $10.24 \pm 3.37$ vs. $2.35 \pm 1.10 \%$; WBC, $19.26 \pm 2.55$ vs. $5.46 \pm 1.41 \%$; $\mathrm{P}<0.05$ for all cell types; Fig. 1A). The percentage of $\mathrm{PS}^{+} \mathrm{ECs}$ after treatment with patient serum was 4.30 -fold higher than after treatment with healthy plasma $(\mathrm{P}<0.05$; Fig. $1 \mathrm{~B})$. The absolute number of $\mathrm{PS}^{+}$blood cells was also significantly higher in patients with MM compared with healthy subjects $(\mathrm{P}<0.05$, for all; Table II). To observe PS binding on the outer membrane of cells, erythrocytes, platelets, or leukocytes were incubated with Alexa Fluor 488-lactadherin and PI, and imaged using confocal microscopy. Alexa Fluor 488-lactadherin staining was not observed on the membranes of platelets, erythrocytes and leukocytes from healthy subjects (Fig. 1C), whereas light green fluorescence was observed on platelets, leukocytes and erythrocytes from patients with MM (Fig. 1D). These results further confirmed that there is increased PS exposure on blood cells in patients with MM compared with healthy subjects.

ECs incubated for $24 \mathrm{~h}$ with serum from patients with MM or healthy control were stained with CD31-Alexa Fluor 647 and PS exposure was detected using lactadherin-Alexa Fluor 488. Confocal laser-scanning microscopy demonstrated that there was limited lactadherin staining on ECs cultured with normal serum (Fig. 1C), while large amounts of lactadherin, exhibited
Table II. Absolute number of PS-positive blood cells in study subjects.

\begin{tabular}{lcc}
\hline PS $^{+}$blood cells & Healthy subjects & Multiple myeloma \\
\hline Erythrocyte $\left(\times 10^{10} / 1\right)$ & $2.20 \pm 1.30$ & $3.71 \pm 5.93^{\mathrm{a}}$ \\
Platelet $\left(\times 10^{9} / 1\right)$ & $5.33 \pm 2.96$ & $19.24 \pm 9.69^{\mathrm{a}}$ \\
Leukocytes $\left(\mathrm{x} 10^{8} / 1\right)$ & $3.16 \pm 0.93$ & $10.23 \pm 3.34^{\mathrm{a}}$
\end{tabular}

${ }^{\mathrm{a}} \mathrm{P}<0.05$ vs. healthy subjects. Data are presented as the mean \pm standard deviation. PS, phosphatidylserine.

by green fluorescence, was observed on ECs treated with patient serum (Fig. 1D). Notably, the percentage of $\mathrm{PS}^{+} \mathrm{ECs}$ was higher than that of erythrocytes, platelets and leukocytes in patients with MM.

Coagulation time and inhibition assay. To examine whether the increase in $\mathrm{PS}^{+}$cells contributes to enhanced PCA, recalcification-time assays were performed using a KC4A-coagulometer. Compared with samples from healthy subjects, patient suspensions of erythrocytes, platelets and leukocytes (cell number controlled) exhibited significantly shorter coagulation time $(\mathrm{P}<0.05$; Fig. 2A). ECs cultured in serum from patients with $\mathrm{MM}$ induced a shorter coagulation time when cultured in 

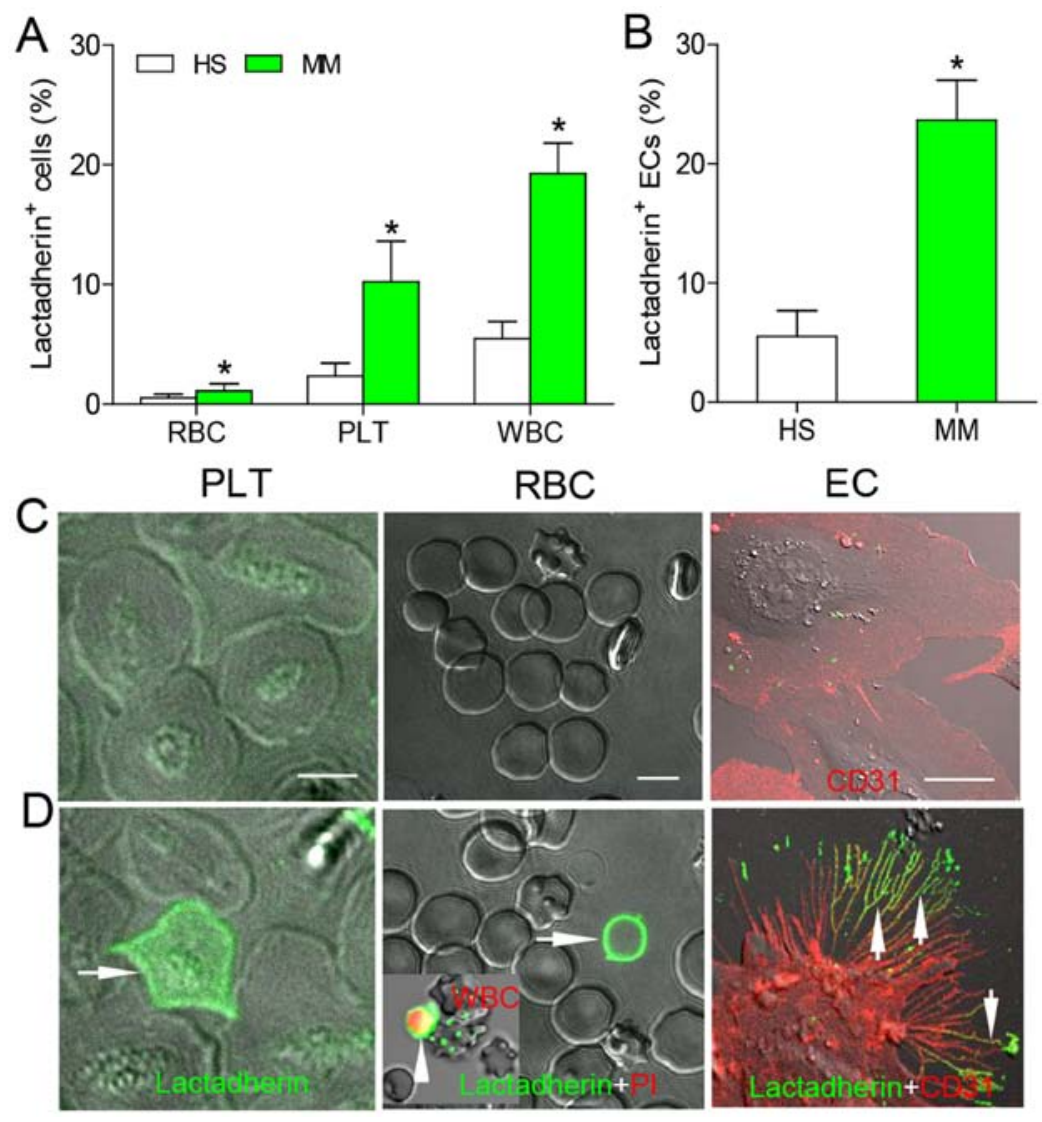

Figure 1. PS exposure on blood cells and HUVECs was analyzed by flow cytometry and confocal microscopy. Blood cells of (A) HS and patients with MM and (B) HUVECs treated with HS or MM serum were incubated with Alexa 488-conjugated lactadherin and assessed by flow cytometry. Data are presented as the mean \pm standard deviation, ${ }^{*} \mathrm{P}<0.05$ vs. HS. Alexa Fluor 488 -lactadherin staining was performed on blood samples from (C) HS and (D) patients with MM; almost no staining was observed on cells from HS; WBCs were identified using PI staining (red fluorescence, inset). ECs with (C) HS or (D) patient serum were stained with CD31-Alexa Fluor 647 and PS exposure was detected using lactadherin-Alexa Fluor 488 at 24-h incubation; almost no lactadherin staining was observed on normal HUVECs. Treatment of HUVECs with patient serum led to retraction of cell margins, extension of filopods, and lactadherin (green) binding on filopods (arrows); scale bar, $5 \mu \mathrm{m}$. PS, phosphatidylserine; HUVEC, human umbilical vein endothelial cells; HS, healthy subjects; MM, multiple myeloma; PLT, platelets; RBCs, red blood cells (erythrocytes); EC, endothelial cell; WBCs, white blood cells (leukocytes); PI, propidium iodide.
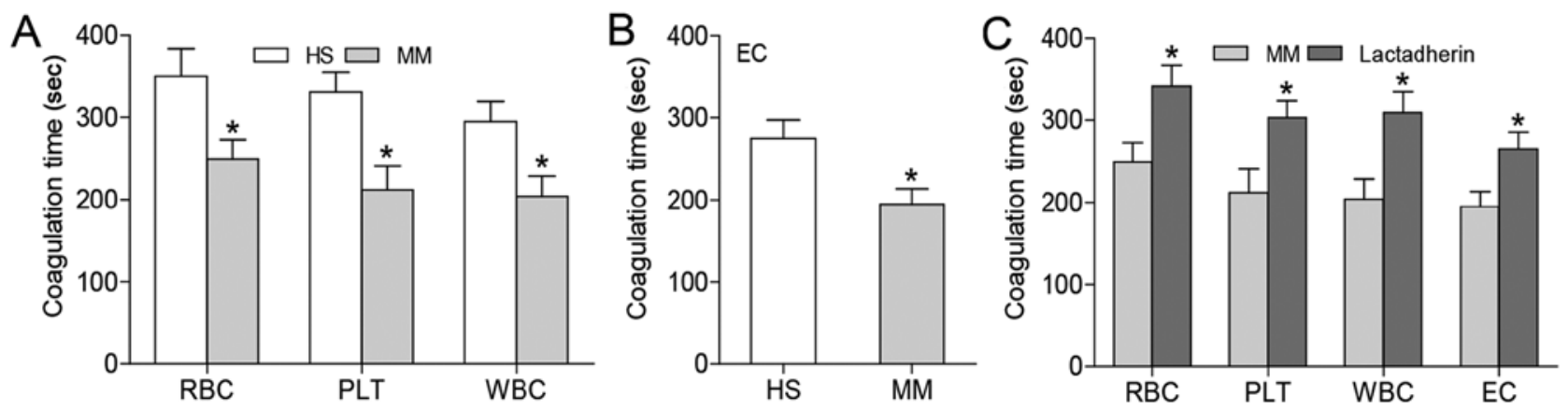

Figure 2. Coagulation time and inhibition assay. (A) Coagulation times of RBCs, PLTs, or WBCs from healthy subjects and patients with MM were evaluated. Blood cells of MM patients had more procoagulant activity than those from healthy subjects. (B) ECs cultured with patient serum for $24 \mathrm{~h}$ showed more PCA than those cultured with healthy serum. (C) Procoagulant activity of blood cells from MM patients and cultured ECs in patient serum for $24 \mathrm{~h}$ was detected in the absence or presence of lactadherin $(128 \mathrm{nM})$; lactadherin entirely inhibited procoagulant activity induced by blood cells and ECs. Data are representative of triplicate independent experiments, and are displayed as the mean \pm standard deviation. ${ }^{*} \mathrm{P}<0.05$ vs. HS. HS, healthy subjects; MM, multiple myeloma; RBCs, red blood cells (erythrocytes); PLT, platelets; WBCs, white blood cells (leukocytes); EC, human umbilical vein endothelial cells.

serum from healthy subjects $(\mathrm{P}<0.05$; Fig. 2B). To confirm whether this increased PCA was attributed to membrane PS exposure, inhibition assays were performed where cells were incubated with $128 \mathrm{nM}$ lactadherin prior to coagulation testing. Lactadherin prolonged the coagulation time of $\mathrm{PS}^{+}$cells to similar values as healthy subjects $(\mathrm{P}<0.05$; Fig. $2 \mathrm{C})$.
Role of PS in formation of procoagulant enzyme complexes. In order to further evaluate the capacity of blood cells and ECs to support the formation of procoagulant enzyme complexes, intrinsic/extrinsic factor Xa and thrombin formation assays were performed using purified coagulation factors. The production of all three procoagulant enzyme complexes was 
A

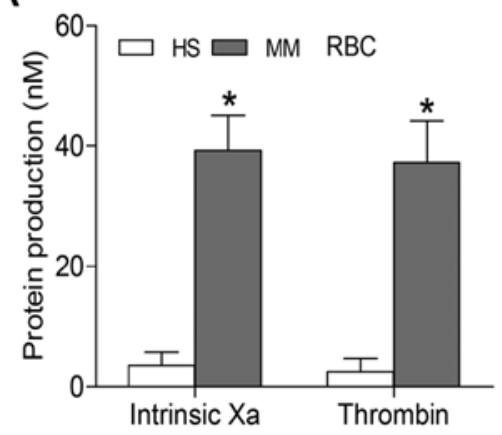

B

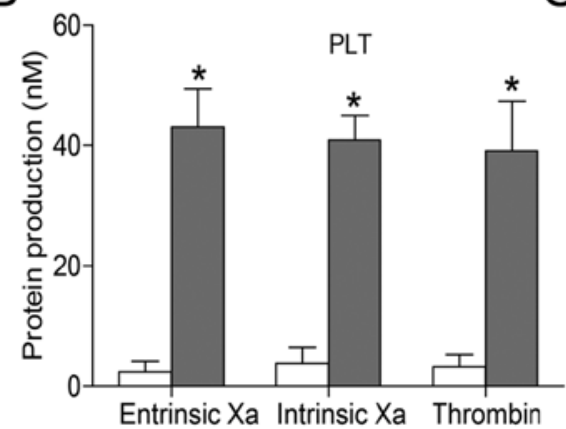

C

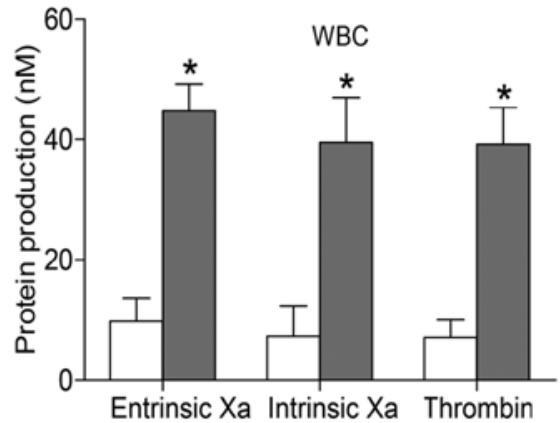

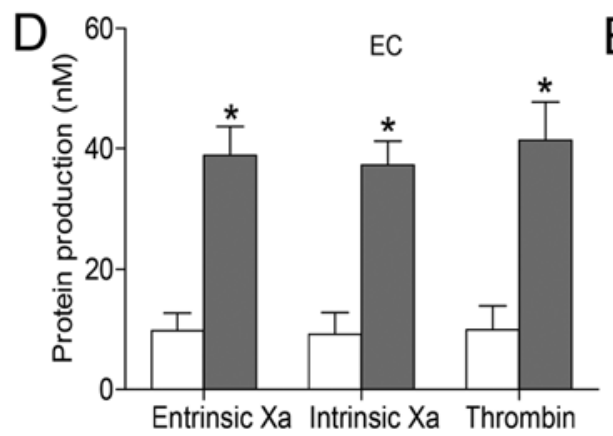

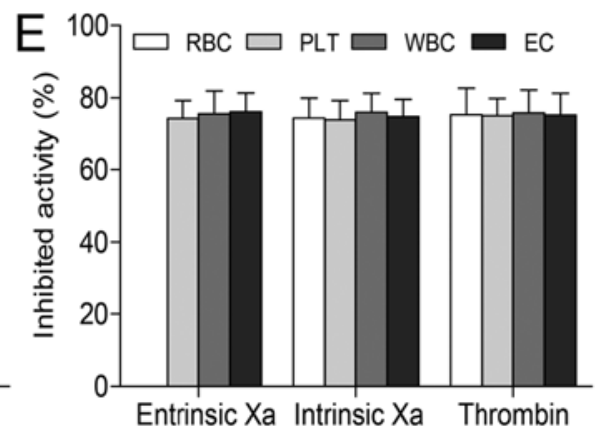

Figure 3. Formation and inhibition assays of procoagulant enzyme complexes. Xa and thrombin production in the presence of (A) $2 \times 10^{5}$ RBCs, (B) $2 \times 10^{4}$ PLTs or (C) $2 \times 10^{4} \mathrm{WBC}$ from healthy subjects and patients with MM. Experiments were also performed using (D) $2 \times 10^{4}$ ECs cultured with normal or patient serum for $24 \mathrm{~h}$. Intrinsic Xa formation was measured in the presence of intrinsic Xa, FVIII and thrombin. Extrinsic Xa production was assessed in the presence of FVIIa. Thrombin generation was investigated in the presence of Xa and FVa. (E) Capacity of lactadherin (128 nM) to block procoagulant enzyme complexes on cells from patients with MM was evaluated; in each cell type, lactadherin decreased activity of the procoagulant enzyme complexes by $75 \%$. Results displayed as the mean \pm standard deviation, ${ }^{*} \mathrm{P}<0.05$ vs. HS. FVIII, factor VIII; FVa, factor Va; HS, healthy subjects; MM, multiple myeloma; RBCs, red blood cells (erythrocytes); Xa, factor Xa; PLT, platelets; WBCs, white blood cells (leukocytes); EC, human umbilical vein endothelial cells.

A

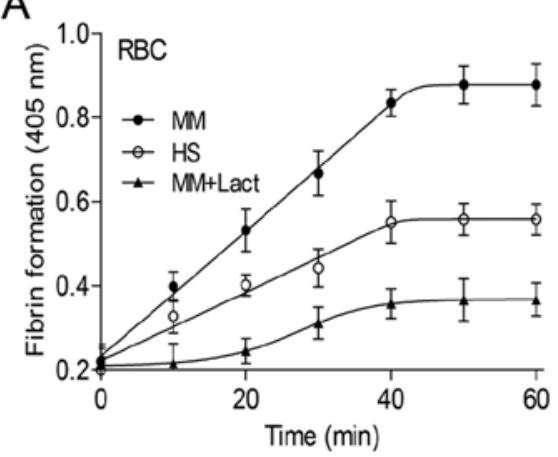

B

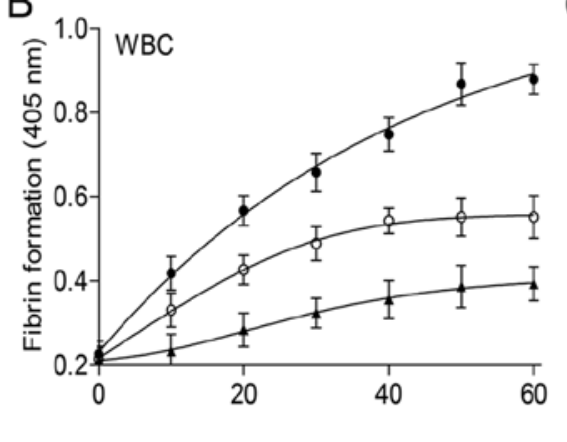

C

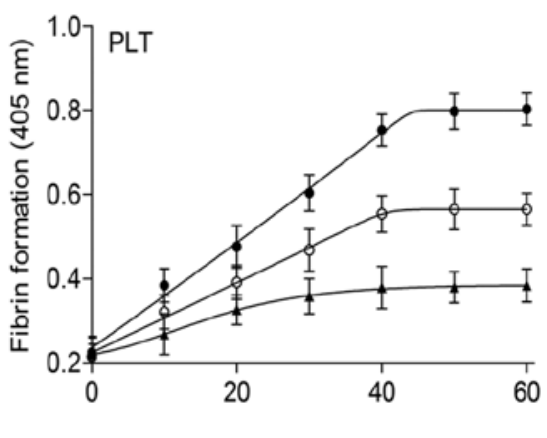

Figure 4. Fibrin formation and inhibition assays. Fibrin production supported by blood cells from healthy subjects and patients with MM was measured by turbidity. (A) RBCs, (B) WBCs and (C) PLTs from patients with MM supported more fibrin generation than those from HS. Preincubation with lactadherin $(128 \mathrm{nM})$ reduced fibrin generation on blood cells from patients with MM to lower than HS. Data are presented as the mean \pm standard deviation. RBCs, red blood cells (erythrocytes); HS, healthy subjects; MM, multiple myeloma; Lact, lactadherin; WBCs, white blood cells (leukocytes); PLT, platelets.

increased in MM groups compared with healthy subjects $\left(\mathrm{P}<0.05\right.$; Fig. 3A-D). These findings suggest that $\mathrm{PS}^{+}$blood cells contribute to coagulation, and that the relative number of these blood cells may increase thrombosis risk. In inhibition assays for all cell types, lactadherin (at $128 \mathrm{nM}$ ) blocked production of the three procoagulant enzyme complexes by $\sim 75 \%$ (Fig. 3E). PS blockade almost entirely inhibited the formation of these complexes, suggesting that PS independently increases PCA in patients with MM.

Role of PS in fibrin formation on blood cells. Fibrin constitutes the primary structural protein of blood clots and intravascular thrombi and its formation involves the concerted action of coagulation factors and blood cells. Therefore, fibrin formation was evaluated in normal MDP. More fibrin was deposited on erythrocytes, platelets and leukocytes from patients with MM than those from healthy subjects $(\mathrm{P}<0.05$; Fig. 4). The inhibition assays demonstrated that lactadherin reduced fibrin formation of all cells to a level lower than healthy subjects.

Factor Xa/factor Va binding and fibrin deposition around the PS on ECs. Confocal microscopy provided more evidence that PS on ECs has an important role in fibrin clot formation. Limited factor $\mathrm{Va}$ (red) or factor Xa (green) binding was 


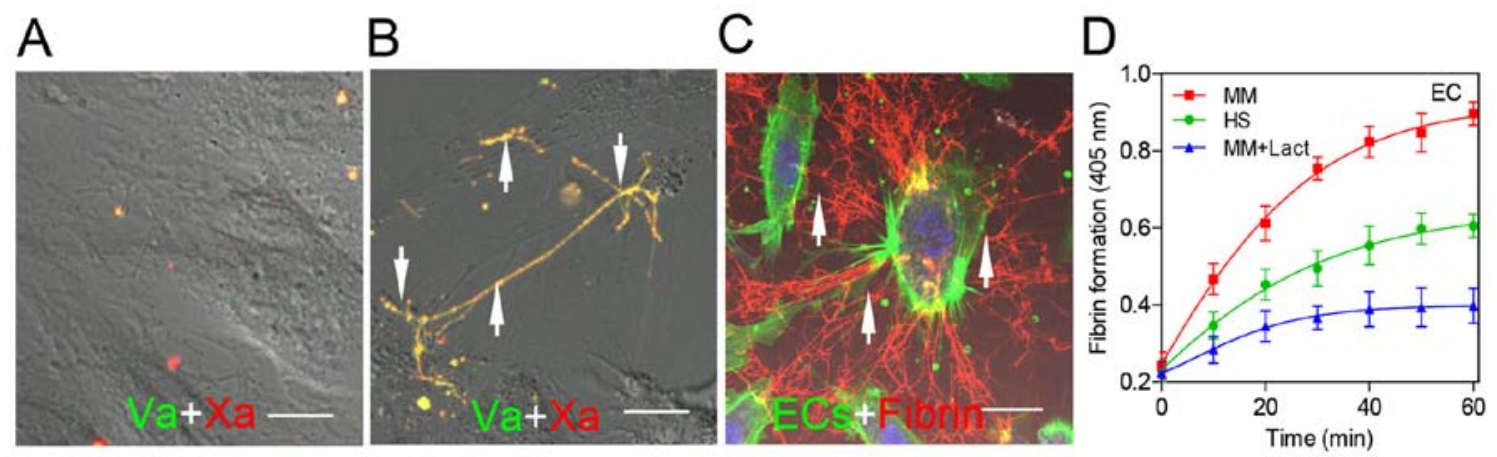

Figure 5. FVa/FXa binding and fibrin deposition on ECs treated with patient serum. FVa/FXa binding on cultured ECs treated with (A) normal serum and (B) serum from patients with MM, detected using confocal microscopy; more FVa and FXa colocalization (yellow) is observed on filopods (arrows) of near the retracted edges of ECs treated with patient serum than normal serum. (C) Cultured ECs were incubated with plasma in the presence of calcium and Alexa 647-conjugated anti-fibrin at $37^{\circ} \mathrm{C}$ for $15 \mathrm{~min}$; ECs and nuclei were visualized by actin (green) staining with fluorescein isothiocyanate-phalloidin and DAPI (blue) and marked fibrin strands were observed along the filopodia of cultured ECs (arrow); scale bar, $2 \mu \mathrm{m}$. (D) Fibrin production on ECs cultured with patient serum was detected in the presence of recalcified MDP with or without $128 \mathrm{nM}$ lactadherin. Data are presented as the mean \pm standard deviation. ECs, human umbilical vein endothelial cells; Va, factor Va; Xa, factor Xa; MM, multiple myeloma; HS, healthy subjects; Lact, lactadherin.
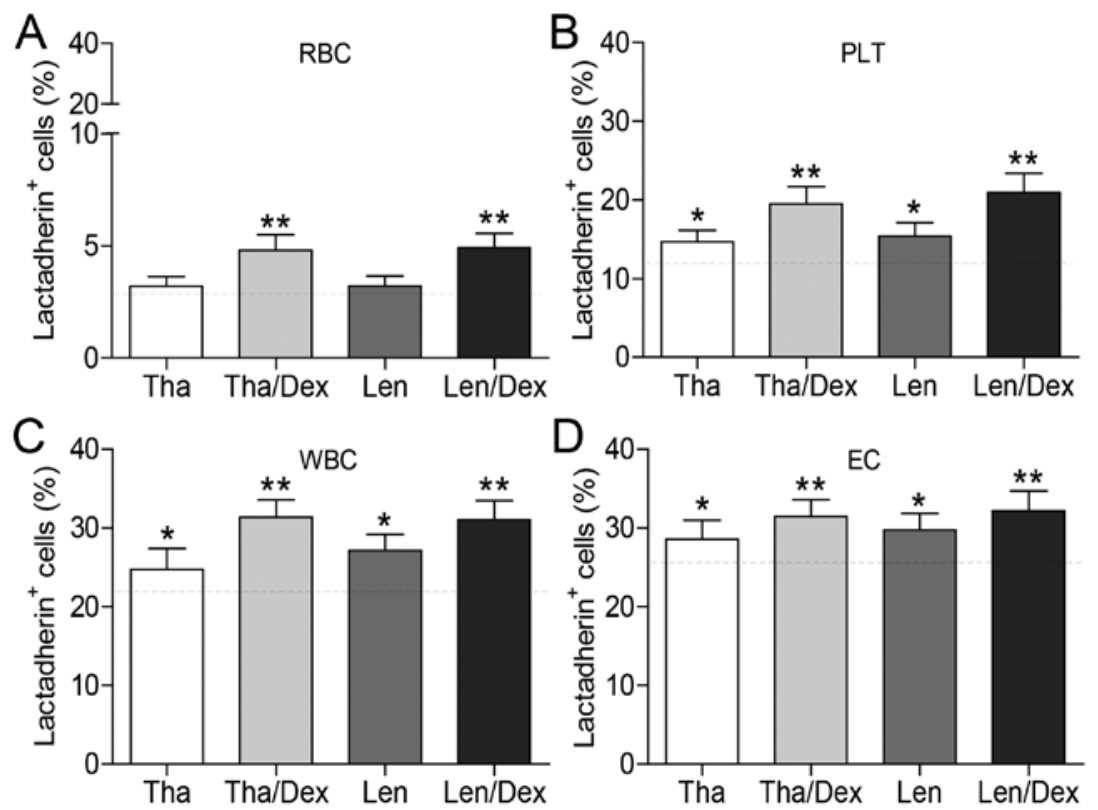

Figure 6. Effect of immunomodulatory drugs-based treatments on PS exposure of blood cells and ECs. (A) RBCs (24 h), (B) PLTs (1 h), (C) WBCs (24 h) from patients with MM were incubated with dimethyl sulfoxide $(0.01 \%)$, Tha $(1.0 \mu \mathrm{M})$, Len $(1.0 \mu \mathrm{M})$, Tal $(1.0 \mu \mathrm{M}) / \operatorname{Dex}(10 \mu \mathrm{M})$, or Len $(1.0 \mu \mathrm{M}) / \operatorname{Dex}(10 \mu \mathrm{M})$ at $37^{\circ} \mathrm{C}$. (D) ECs were incubated in five groups containing $20 \%$ pooled serum obtained from patients with MM at room temperature for $24 \mathrm{~h}$. The gray dashed line represents PS exposure with no drug treatment as control. PS exposure was measured as the \% of cells that were positive for Alexa Fluor 488-lactadherin using flow cytometry. Data are presented as the mean \pm standard deviation, ${ }^{*} \mathrm{P}<0.05$ vs. control, ${ }^{* *} \mathrm{P}<0.05$ vs. Tha or Len. PS, phosphatidylserine; MM, multiple myeloma; RBC, red blood cells (erythrocytes); Tha, thalidomide; Len, lenalidomide; Dex, dexamethasone; PLT, platelets; WBC, WBCs, white blood cells (leukocytes); EC, human umbilical vein endothelial cells.

observed on ECs treated with serum from healthy subjects (Fig. 5A, arrow). When ECs were cultured with the serum from patients with MM, factor Va and factor Xa were observed to be bound to the PS-enriched areas of the cell membrane (Fig. 5B, arrow). ECs were incubated with plasma in the presence of calcium and Alexa 647-conjugated anti-fibrin. Considerable fibrin fibrils (red) were spread along the filopodia of ECs visualized using actin and DAPI staining (Fig. 5C, arrow). The patient serum resulted in a 1.48 -fold higher fibrin generation in ECs than the serum from healthy subjects $(\mathrm{P}<0.05$; Fig. 5D).

Effects of IMiDs with or without Dex to PS exposure on blood cells and ECs. The effects of the IMiDs (Tha and Len, with or without Dex treatment) on PS exposure of blood cells in vitro were determined. Erythrocytes $(24 \mathrm{~h})$, platelets $(1 \mathrm{~h})$, and leukocytes $(24 \mathrm{~h})$ from patient with MM were cultured with Tha $(1.0 \mu \mathrm{M})$, Len $(1.0 \mu \mathrm{M})$, Tha $(1.0 \mu \mathrm{M}) / \operatorname{Dex}(10 \mu \mathrm{M})$, or Len $(1.0 \mu \mathrm{M}) / \operatorname{Dex}(10 \mu \mathrm{M})$ at $37^{\circ} \mathrm{C}$. Flow cytometry analysis demonstrated that, compared with control or either IMiD alone, Tha or Len plus Dex increased the level of PS exposure on erythrocytes $(\mathrm{P}<0.05$; Fig. $6 \mathrm{~A})$. IMiD treatment alone did not significantly increase PS exposure on erythrocytes compared with controls. However, Tha and Len significantly increased PS exposure on platelets and leukocytes compared with controls $(\mathrm{P}<0.05$; Fig. 6B and C). Treatment of platelets and leukocytes with an IMiD in combination with Dex resulted in higher PS 
exposure compared with IMiD treatment alone. The effects of IMiDs and Dex, either alone or in combination, on PS exposure on ECs were determined by flow cytometry. Incubation of ECs with an IMiD alone resulted in increased PS exposure compared with controls $(\mathrm{P}<0.05$; Fig. 6D). Furthermore, an increased percentage of $\mathrm{PS}^{+} \mathrm{ECs}$ was induced by the combination of an IMiD and Dex, as compared with Tha or Len alone. The results indicate that IMiDs and Dex used in combination can further increase PS exposure on blood cells from MM patients and ECs in vitro.

\section{Discussion}

The findings of the current study demonstrated that $\mathrm{PS}^{+}$blood cells may potentially have a role in the induction of a hypercoagulable state in patients with $\mathrm{MM}$, and that patient serum components can induce PS exposure in cultured ECs. Compared with healthy subjects, patients with MM had markedly higher levels of $\mathrm{PS}^{+}$blood cells, and serum from patients with MM increased $\mathrm{PS}^{+}$cultured ECs compared with healthy serum. In addition, the percentage of $\mathrm{PS}^{+} \mathrm{ECs}$ was higher than that of erythrocytes, platelets and leukocytes in patients with MM. Exposed PS contributes to tenase and prothrombinase complex formation, leading to a shorter coagulation time and greater intrinsic/extrinsic factor $\mathrm{Xa}$, thrombin and fibrin generation. In addition, combined treatment with IMiDs plus Dex induced greater PS exposure on platelets, leukocytes and erythrocytes than treatment with IMiDs alone. Finally, lactadherin inhibited production of all three procoagulant enzyme complexes by $\geq 75 \%$, further confirming the procoagulant role of PS in patients with MM.

Although platelet dysfunction often occurs in MM (27), it remains unclear whether platelets contribute to thrombosis in patients with MM. To the best of our knowledge, this is the first study evaluating the role of $\mathrm{PS}^{+}$platelets in the prothrombotic state in patients with MM. The percentage of $\mathrm{PS}^{+}$platelets was significantly higher in patients with MM than in healthy subjects, which indicates abnormal activation or apoptosis of platelets. In a previous study, the serum level of platelet factor 4 was significantly elevated in patients with MM (15), supporting this finding. Notably, $\mathrm{PS}^{+}$platelets had the capacity to increase tenase and prothrombinase complex formation, leading to greater factor Xa, thrombin and fibrin generation. Activated platelets release thrombin leading to platelet aggregation and three-dimensional clot formation (28). Thus, we hypothesize that $\mathrm{PS}^{+}$platelets may recruit more circulating platelets by producing thrombin. On this basis, platelet PS exposure is an important mediator of the hypercoagulable state in patients with MM.

Clinical studies have reported that activated leukocytes are associated with venous thrombosis (29). However, the pathogenic pathways linking leukocyte abnormalities to thrombosis in patients with MM remain unclear. The results of the current study indicated that injured or activated leukocytes increased PCA through PS exposure. This process increases the serum concentration of three procoagulant enzyme complexes by $\mathrm{PS}^{+}$leukocyte-derived PCA and potentially forms the nidus upon which the thrombus develops. Typically, erythrocytes are considered as passive participants in coagulation, merely providing bulk material for the obstructive clot. The observa- tions in the current study provide evidence that erythrocytes may be actively involved in the hypercoagulable state of patients with MM. PS exposure on erythrocytes provides a catalytic surface to support the assembly of blood coagulation factors, thus promoting the coagulation cascade activation and thrombin generation (10). Previous studies reported that $\mathrm{PS}^{+}$erythrocytes are also more adhesive to endothelial cells and prone to form erythrocyte aggregates in chronic uremia and obesity, supporting the conclusions of the present study $(30,31)$.

To investigate the role of vascular endothelium dysfunction in MM-associated thrombosis, cultured ECs were incubated with patient serum, which resulted in a significant increase in PS exposure compared with incubation in serum from healthy subjects. A previous study has indicated patients with MM exhibit pathologically enhanced von Willebrand factor, a marker of ECs activation, which is consistent with the findings of the present study (7). In addition to promoting the formation of thrombin, the results of the current study demonstrate that patient serum-treated ECs highly expressed PS, which supported binding of factor Va and factor Xa, and fibrin deposition. Following activation, imbalance or alteration of 'differential expression of procoagulants and anticoagulants in the endothelium' may modulate endothelial thromboresistance from an anticoagulant state into a procoagulant one $(32,33)$. Thus, it seems reasonable to hypothesize that PS has a pivotal role in the localized procoagulant phenotype of ECs by bringing clotting factors together, resulting in fibrin formation.

It was previously reported that patients with MM have higher levels of endogenous thrombin potential in a global assay of thrombin generation (34). The data of the current study demonstrated that $\mathrm{PS}^{+}$blood cells and ECs from patients with MM supported the formation of intrinsic/extrinsic factor Xase and prothrombinase, essential components of the coagulation cascade that lead to increased thrombin generation. In addition, $\mathrm{PS}^{+}$cells from patients with MM have a shorter coagulation time and higher production of fibrin than samples from healthy subjects. Blockade of PS with lactadherin prolonged coagulation time and decreased fibrin formation to control levels and inhibited $\sim 75 \%$ of the procoagulant enzyme production. By contrast, in a previous study, anti-tissue factor antibody had a negligible effect on PCA of cells from patients with MM; this may be explained by the fact that plasma exposed tissue factor is generally quiescent, with little or no detectable PCA, unless it resides in a membrane containing PS $(35,36)$. The results indicate that increased PS contributes to the hypercoagulable state in patients with MM.

The exact mechanism of the increased risk of VTE in patients with MM treated with IMiDs-based regimens is not yet fully understood. The results of the current study have demonstrated that treatment with IMiDs plus Dex induces higher levels of PS ${ }^{+}$blood cells and ECs than controls or IMiDs alone, in vitro. IMiDs with Dex has been previously reported to increase P-selectin expression on platelets and induce EC injury, supporting the findings of the present study $(37,38)$. In addition, IMiDs stimulate T-cell production and activation of natural killer cells (39). Thus, we hypothesize that treatment with Dex may aggravate the abnormal activation or apoptosis of blood cells in patients with MM using IMiDs-based regimens. Considering the high cost of treatment (40), the number 
of patients that receive IMiDs-based regimens is low in China. The in vivo effect of IMiDs-based regimens on PCA of blood cells and ECs from patients with MM will be investigated in future studies.

In summary, $\mathrm{PS}^{+}$blood cells and PS-associated PCA were increased in the circulation of patients with $\mathrm{MM}$ and patient serum induced PS exposure on cultured ECs. In addition, IMiDs-based treatment increased PS exposure on blood cells and ECs in vitro. Increasing levels of $\mathrm{PS}^{+}$cells were associated with a hypercoagulable state in patients with MM. Furthermore, PS inhibition assays using lactadherin suggest that blockage of PS may constitute a novel therapy for preventing thrombosis. Future clinical trials are required to investigate this hypothesis.

\section{Acknowledgements}

Not applicable.

\section{Funding}

This study was supported by grants from the National Natural Science Foundation of China (cat. no. 81470301 and 81670128).

\section{Availability of data and materials}

The analyzed data sets generated during the study are available from the corresponding author on reasonable request.

\section{Authors' contributions}

Study concept and design (LG, JS); data acquisition, clinical data and sample collection, statistical analysis (LG, DT, MY, CW, PZ, JJ, BL, YL, RL); data interpretation and manuscript drafting (LG, JS); critical revision of the manuscript (LG, VN, DT, MY, YZ, TL, JS); YZ, TL, ZD, YT, JZ, YB and JK provided valuable advice for this study. All authors have read and approved the final manuscript.

\section{Ethics approval and consent to participate}

Harbin Medical University Ethical Committee (Harbin, China) approved the study, and patients provided written, informed consent.

\section{Consent for publication}

Not applicable.

\section{Competing interests}

JS has a patent for the use of lactadherin as a probe for phosphatidylserine. Other authors declare that they have no competing interests.

\section{References}

1. Kristinsson SY, Pfeiffer RM, Björkholm M, Goldin LR, Schulman S, Blimark C, Mellqvist UH, Wahlin A, Turesson I and Landgren O: Arterial and venous thrombosis in monoclonal gammopathy of undetermined significance and multiple myeloma: A population-based study. Blood 115: 4991-4998, 2010.
2. Blom JW, Doggen CJ, Osanto S and Rosendaal FR: Malignancies, prothrombotic mutations, and the risk of venous thrombosis. JAMA 293: 715-722, 2005.

3. Kristinsson SY, Pfeiffer RM, Björkholm M, Schulman S and Landgren O: Thrombosis is associated with inferior survival in multiple myeloma. Haematologica 97: 1603-1607, 2012.

4. Palumbo A, Rajkumar SV, Dimopoulos MA, Richardson PG, San Miguel J, Barlogie B, Harousseau J, Zonder JA, Cavo M, Zangari $\mathrm{M}$, et al; International Myeloma Working Group: Prevention of thalidomide- and lenalidomide-associated thrombosis in myeloma. Leukemia 22: 414-423, 2008.

5. De Stefano V, Za T and Rossi E: Venous thromboembolism in multiple myeloma. Semin Thromb Hemost 40: 338-347, 2014.

6. Auwerda JJ, Sonneveld P, de Maat MP and Leebeek FW: Prothrombotic coagulation abnormalities in patients with newly diagnosed multiple myeloma. Haematologica 92: 279-280, 2007.

7. Minnema MC, Fijnheer R, De Groot PG and Lokhorst HM: Extremely high levels of von Willebrand factor antigen and of procoagulant factor VIII found in multiple myeloma patients are associated with activity status but not with thalidomide treatment. J Thromb Haemost 1: 445-449, 2003.

8. Elice F, Fink L, Tricot G, Barlogie B and Zangari M: Acquired resistance to activated protein $\mathrm{C}$ (aAPCR) in multiple myeloma is a transitory abnormality associated with an increased risk of venous thromboembolism. Br J Haematol 134: 399-405, 2006.

9. van Marion AM, Auwerda JJ, Minnema MC, van Oosterom R, Adelmeijer J, de Groot PG, Leebeek FW, Sonneveld P, Lokhorst HM and Lisman T: Hypofibrinolysis during induction treatment of multiple myeloma may increase the risk of venous thrombosis. Thromb Haemost 94: 1341-1343, 2005.

10. Zwaal RF and Schroit AJ: Pathophysiologic implications of membrane phospholipid asymmetry in blood cells. Blood 89: 1121-1132, 1997.

11. Vance JE and Steenbergen R: Metabolism and functions of phosphatidylserine. Prog Lipid Res 44: 207-234, 2005.

12. Gao C, Xie R, Yu C, Wang Q, Shi F, Yao C, Xie R, Zhou J, Gilbert GE and Shi J: Procoagulant activity of erythrocytes and platelets through phosphatidylserine exposure and microparticles release in patients with nephrotic syndrome. Thromb Haemost 107: 681-689, 2012.

13. Zhou J, Shi J, Hou J, Cao F, Zhang Y, Rasmussen JT, Heegaard CW and Gilbert GE: Phosphatidylserine exposure and procoagulant activity in acute promyelocytic leukemia. J Thromb Haemost 8: 773-782, 2010

14. Jurczyszyn A, Czepiel J, Biesiada G, Gdula-Argasińska J, Cibor D, Owczarek D, Perucki W and Skotnicki AB: HGF, sIL-6R and TGF- $\beta 1$ play a significant role in the progression of multiple myeloma. J Cancer 5: 518-524, 2014.

15. Fritz E, Ludwig H, Scheithauer W and Sinzinger H: Shortened platelet half-life in multiple myeloma. Blood 68: 514-520, 1986.

16. Lee H, Kong SY, Sohn JY, Shim H, Youn HS, Lee S, Kim HJ and Eom HS: Elevated red blood cell distribution width as a simple prognostic factor in patients with symptomatic multiple myeloma. Biomed Res Int 2014: 145619, 2014.

17. Kerr R, Stirling D and Ludlam CA: Interleukin 6 and haemostasis. Br J Haematol 115: 3-12, 2001.

18. Mechtcheriakova D, Schabbauer G, Lucerna M, Clauss M, De Martin R, Binder BR and Hofer E; De Martin R: Specificity, diversity, and convergence in VEGF and TNF-alpha signaling events leading to tissue factor up-regulation via EGR-1 in endothelial cells. FASEB J 15: 230-242, 2001.

19. Hoshi A, Matsumoto A, Chung J, Isozumi Y and Koyama T: Activation of coagulation by a thalidomide-based regimen. Blood Coagul Fibrinolysis 22: 532-540, 2011.

20. Isozumi Y, Arai R, Fujimoto K and Koyama T: Activation of coagulation by lenalidomide-based regimens for the treatment of multiple myeloma. PLoS One 8: e64369, 2013.

21. Shi J, Shi Y, Waehrens LN, Rasmussen JT, Heegaard CW and Gilbert GE: Lactadherin detects early phosphatidylserine exposure on immortalized leukemia cells undergoing programmed cell death. Cytometry A 69: 1193-1201, 2006.

22. Rajkumar SV, Dimopoulos MA, Palumbo A, Blade J, Merlini G, Mateos MV, Kumar S, Hillengass J, Kastritis E, Richardson P, et al: International Myeloma Working Group updated criteria for the diagnosis of multiple myeloma. Lancet Oncol 15: e538-e548, 2014.

23. Lang E, Gatidis S, Freise NF, Bock H, Kubitz R, Lauermann C, Orth HM, Klindt C, Schuier M, Keitel V, et al: Conjugated bilirubin triggers anemia by inducing erythrocyte death. Hepatology 61: 275-284, 2015. 
24. NaveenKumar SK, Thushara RM, Sundaram MS, HemshekharM, Paul M, Thirunavukkarasu C, Basappa, Nagaraju G, Raghavan SC, Girish KS, et al: Unconjugated bilirubin exerts pro-apoptotic effect on platelets via p38-MAPK activation. Sci Rep 5: 15045, 2015.

25. Khan NM and Poduval TB: Immunomodulatory and immunotoxic effects of bilirubin: Molecular mechanisms. J Leukoc Biol 90: 997-1015, 2011.

26. Campbell RA, Overmyer KA, Selzman CH, Sheridan BC and Wolberg AS: Contributions of extravascular and intravascular cells to fibrin network formation, structure, and stability. Blood 114: 4886-4896, 2009.

27. Eby C: Pathogenesis and management of bleeding and thrombosis in plasma cell dyscrasias. Br J Haematol 145: 151-163, 2009.

28. Koupenova M, Kehrel BE, Corkrey HA and Freedman JE: Thrombosis and platelets: An update. Eur Heart J 38: 785-791, 2017.

29. Swystun LL and Liaw PC: The role of leukocytes in thrombosis. Blood 128: 753-762, 2016.

30. Bonomini M, Sirolli V, Gizzi F, Di Stante S, Grilli A and Felaco M: Enhanced adherence of human uremic erythrocytes to vascular endothelium: Role of phosphatidylserine exposure. Kidney Int 62: 1358-1363, 2002.

31. Solá E, Vayá A, Martínez M, Moscardó A, Corella D, Santaolaria ML, España F and Hernández-Mijares A: Erythrocyte membrane phosphatidylserine exposure in obesity. Obesity (Silver Spring) 17: 318-322, 2009.

32. Aird WC: Phenotypic heterogeneity of the endothelium: II Representative vascular beds. Circ Res 100: 174-190, 2007.
33. Levi M, Nieuwdorp M, van der Poll T and Stroes E: Metabolic modulation of inflammation-induced activation of coagulation. Semin Thromb Hemost 34: 26-32, 2008.

34. Petropoulou AD, Gerotziafas GT, Samama MM, Hatmi M, Rendu F and Elalamy I: In vitro study of the hypercoagulable state in multiple myeloma patients treated or not with thalidomide. Thromb Res 121: 493-497, 2008.

35. Cimmino G, Ciccarelli G and Golino P: Role of tissue factor in the coagulation network. Semin Thromb Hemost 41: 708-717, 2015.

36. Chen VM and Hogg PJ: Encryption and decryption of tissue factor. J Thromb Haemost 11 (Suppl 1): 277-284, 2013.

37. Dunkley S and Gaudry L: Thalidomide causes platelet activation, which can be abrogated by aspirin. J Thromb Haemost 5: 1323-1325, 2007

38. Rosovsky R, Hong F, Tocco D, Connell B, Mitsiades C, Schlossman R, Ghobrial I, Lockridge L, Warren D, Bradwin G, et al: Endothelial stress products and coagulation markers in patients with multiple myeloma treated with lenalidomide plus dexamethasone: An observational study. Br J Haematol 160: 351-358, 2013.

39. Quach H, Ritchie D, Stewart AK, Neeson P, Harrison S, Smyth MJ and Prince HM: Mechanism of action of immunomodulatory drugs (IMiDS) in multiple myeloma. Leukemia 24: 22-32, 2010.

40. Wang J, Guo H and Zhou X: Clinical utility and patient consideration in the use of lenalidomide for multiple myeloma in Chinese patients. Onco Targets Ther 8: 1277-1284, 2015. 